\title{
Economic policy and statistics
}

\author{
S. L. LOUWES
}

Statistical Office of the European Communities, Brussels, Belgium

\section{Introduction}

The Statistical Office of the European Communities is at present faced with a number of problems concerning the relation between economic policy and statistics.

Some of these problems result from the rapid development of the common agricultural policy, the object of which is to integrate the agricultural economies of the six Member States into one common market, after a transitional period. In many respects this common agricultural policy is more than the sum of its parts, i.e. more than the sum of the separate agricultural policies pursued up till now in the various Member States. As we have said, it envisages the creation of one big common market, and is being carried out with speed and imagination. One of the instruments of this common policy is the national system for collecting agricultural statistics which each of the six Member States contributes. Here, however, the total result is no more than the sum of the parts - in fact it may well be less, because statistics are governed by the laws of inaccurate figures, which virtually amounts to saying that "a chain is no stronger than its weakest link".

On the one hand, then, we have a rapid development of the common agricultural policy which includes a rather detailed organization of the market, and the creation of the policy instruments required (e.g. levy systems, the machinery for intervention, aid funds); and on the other we have statistical offices that are geared to forms of agricultural policy which work on different lines (more quantitative regulation) or are simpler.

This discrepancy between the development of agricultural policy and the situation of agricultural statistics in the EEC automatically raises the question of the connection between policy and statistics. At bottom, the situation is plain and straightforward. In order to carry out an effective policy, the body responsible needs both general and particular information. This information will often be of a very specific character, especially if economic policy is as detailed as it is in the EEC today. The statistician must therefore try to obtain the information required as efficiently and accurately as possible with the means and time available. Thus, at first sight there are no problems. Those responsible for policy say what data are necessary, and the statistician works them out as well as he can. But in practice there are problems and complications, as we shall try to explain in this article. An attempt will be made to cast the entire question in a form which is usual in decision theory, with the whole process of decision-making (viz. establishment of the objectives, examination of the courses by which they could be achieved, and collection of information on the real situation in which the whole process takes place) assembled in one analytical framework, in order to attain the optimum result. Only when the connection between economic policy and statistics is clearly understood will it be possible to lay down rules for further development of the agricultural statistics in the service of the Community's common agricultural policy.

Received for publication 6th June, 1965. 


\section{Decision theory}

Like all other policies, agricultural policy is a body of decisions taken over a period of time. A decision consists in having to make a choice between a number of possible actions. Modern decision theory deals with the making of this choice; it examines the process of decision-making scientifically in order to ascertain which elements must be considered if the choice is to be the best possible one.

This theory has grown mainly from experience and developments in economics and statistics. Only in recent years have people begun to apply a uniform procedure in these two sciences in order to arrive at decisions in situations fraught with uncertainties, and that procedure has been developed into a branch on its own - decision theory.

The theory starts with the decision-maker, i.e. the person or policy-making body wishing to mould the ambient situation in pursuit of a certain aim. If only one action can be taken to achieve this aim, no choice need be made, and consequently no decision either, other than whether to pursue the aim or not. Decision theory is concerned with aims that can be achieved via any one of a number of actions, between which a choice is therefore necessary.

Each decision can be broken down into five elements: -

a. the unit making the decision,

b. the aim, in which the preference of the deciding unit is expressed,

c. the several actions that can be taken to achieve this aim,

d. the situation, on which the decision-maker's activity impinges, and in the context of which the aim must be achieved,

e. the rules and criteria applied to determine the choice.

According to decision theory, the decision-maker must proceed as follows: - First he should ascertain what actions are open to him in the light of his aim. Next he must forecast the consequences of each of them, and evaluate those consequences by reference to his utility function. After that, he must make the best choice, using the criterion selected. Accordingly, the decision process in its simplest form can be reduced to the following phases: -

Phase $l$ - Formulation of all possible operations or actions $\left(a_{1} \ldots a_{i} \ldots a_{n}\right)$.

Phase 2 - Establishment of the possible consequences of these actions $y_{i j}(i=1$, $2 \ldots \mathrm{n} ; \mathrm{j}=1,2 \ldots \mathrm{m})$. In the simplest case, each action has one particular consequence $y_{i}$. In most cases, however, the real situation affected by the decision is not known. During the period to which the decision relates, this reality may be in any of a more or less large number of states. These are called the possible "states of nature", and are generally indicated by $\Theta_{j}(\mathrm{j}=1 \ldots \mathrm{m})$. It is assumed that they are limited in number. The consequences of each action $i$ on each state $j$ $(\mathrm{j}=1,2 \ldots \mathrm{m})$ must be ascertained. In all, therefore, we have $\mathrm{n} \times \mathrm{m}$ possible consequences $\mathrm{y}_{\mathrm{ij}}$.

Phase 3 - Evaluation of each consequence by reference to the decision-maker's utility function. A utility $V\left(y_{i j}\right)$ is assigned to each consequence $y_{\mathrm{ij}}$. This results in a "pay-off matrix"' 1 L $(a, \Theta)$.

1. Matrix $=$ a square table of figures, in this case with $m$ columns and $n$ rows. 
Phase 4 - Finally, choice of the most favourable distribution on the basis of a criterion. The question of which criterion to adopt is an essential element of decision theory. The theory offers various criteria of choice, and it is up to the decision-maker to choose one of them, from which the decision will automatically follow. The decision-maker's choice of criterion is decided primarily by his willingness to take risks, and is dependent on the pay-off matrix $\mathrm{L}(\mathrm{a}, \Theta)$.

Decision-making is usually not so simple as it looks from our description. Complications may arise, especially at three points: -

a. The element of uncertainty regarding the state of "nature". In the simplest case, this state is known to a degree bordering on certainty. In more complicated cases, it is only possible to define, with a high degree of probability, the limits within which the situation will lie.

b. The time element in the decision. Each decision has a different "time horizon", i.e. the period in which it is operative. It may relate to something to be done next minute which will have consequences only on the rest of the day, for instance whether to take the 9.32 or 9.58 train to visit a neighbouring town. But the decision can also relate to something to be done for the next few years which will influence the whole course of somebody's life, for instance choice of a profession, or whether to follow a certain programme of studies. Other examples: each day or each week the Government fixes, as part of its market policy, a minimum price which is valid until the next one is fixed; or it decides to carry out a plan for consolidating agricultural holdings, or a land reclamation programme, which will determine the destiny of a whole area for many years to come.

c. Evaluation of each consequence $y_{i j}$ - a far from simple matter. It is easiest when all possible consequences can be measured in terms of money. It becomes more difficult when they have economic, political and social repercussions, or when the opinions of various people or policy-making organizations come into play. Grading the various alternative consequences by one or more yardsticks is difficult enough for one person; but it is even more difficult if a group of people (together functioning as policy-making organization) have to give the final judgement.

There are already theoretical methods of obtaining exact evaluation, but this does not mean that the problem has been solved. We still have too few cases of actual measurement.

Although it cannot be said that theoretical studies have overcome all these drawbacks, great progress has been made and a kind of analysis model has been devised which can be very useful in taking any decisions. It gives guidance in further investigation of all the factors concerned in such a way that the pros and cons of a decision ultimately become much clearer, with a chance of better decisions.

We shall examine below two of the above three complications: -

A. Uncertainty

The uncertainty regarding the state of a situation has given rise to the following theoretical classification: -

1. Certainty situation. Here, the pay-off matrix is an $n \times 1$ matrix, and the whole problem has been reduced to the pure problem of economic choice. The action chosen will be the one which yields the best all-round result.

2. Game-theory situation. Here, the reality affected by someone's decision is supposed to be an opponent, who will deliberately try to prevent the decision-maker 
from achieving the best possible result. This situation is found in most games, hence the name. Its simplest form is that of the zerosum game for two players, each of whom is allowed to make a number of moves. As each player moves, the amount due from one to the other is found in the appropriate pay-off matrix. Since one wins what the other loses, they are uncompromising antagonists, and each will ensure that the other has no chance of "seeing his cards". On the theoretical plane, a solution to this zero-sum game for two players has been elaborated in the sense that for each player one way of playing has been worked out which will produce the best possible result for him. No such solution has been found for games involving more than two persons and a non-zero-sum pay-off matrix.

3. Decision-theory situation. It is often unnecessary to assume that the decisionmaker's adversary will directly attack him and try to take advantage of his mistakes. This situation has been called playing against Nature, on the analogy of the theory of games. Two cases are possible: -

3.1. Prior-probability distribution over the possible states of reality. What Nature is going to do is not exactly known, but some idea can be got from past experience or from information that someone can give. A weighted average will be calculated for the whole series of consequences of one action, in which probabilities will be taken as weighting coefficients. The result is a single series of weighted averages, from which a choice can be made as described under 1 .

3.2. No prior-probability distribution. A number of different selection criteria have been proposed for this situation, but as it lies outside the scope of the article, the reader may be referred to the relevant literature ${ }^{1}$.

4. Statistical decision-theory situation. Here a sample may be obtained which can provide further information on the ambient situation and from which a certain probability distribution can be derived. The other player, Nature, allows a glance to be taken at its cards, it being naturally assumed that Nature will not give an erroneous sample to its own detriment. This branch of decision theory is the farthest advanced; one of its achievements has been to improve a number of statistical techniques which have long been known.

As will also be seen from the examples, sampling often enables the element of uncertainty to be considerably reduced, thereby making a better decision possible. The value of such a sample can therefore be deduced from the greater usefulness of the better-based decision.

The most important new element in this approach is that all possible consequences of all possible actions are examined and evaluated, and only then is a judgement passed, by reference to a criterion established beforehand. A well-founded decision can therefore be arrived at. The decision-maker will not be tempted only to look at the highest result under the most favourable circumstances, but will also consider the less favourable repercussions should these most favourable circumstances not arise. The risk of the wrong action is therefore explicitly examined.

B. Ti m e

A decision seldom stands alone. It is usually one of a chain in which each decision is based on the results of its predecessor. The method of learning about a job by

1 For instance W. J. BAUMOL: Economic theory and operation analysis. Prentice-Hall Inc., Englewood Cliffs, N.J., 1961. 
tackling it is one of the most important means available to man for arriving at good decisions, i.e. by carefully following and checking the consequences of his previous decision, and thus building up a store of knowledge which can be used in the forecasting and evaluating phase of his next decision. When action and reaction take place very fast, and a long chain of decisions following close on each other is involved, the rather intricate decision model described above, which is necessary to cope with the uncertainty in the case, can be simplified by carrying out limited actions which appear prima facie to be the best, and watching the reaction. If this is in line with what is wanted but does not go far enough, the action can be stepped up. If however the action fails to achieve its purpose, it can be modified, or other actions chosen. The labour of analysing all possible actions and their consequences is eliminated, because the rapidity of action and reaction greatly reduces the element of uncertainty. A second condition for application of this model is that the consequence of each action is limited and reversible. "Russian roulette" remains a dangerous game, although the time between action and reaction is very short in this case.

To apply this model it is only necessary to know the objective, and the direction in which the various possible actions take effect. This analysis of actions and reactions can often be incorporated automatically in the decision mechanism, giving rise to what is called the "feed-back system". The most frequently quoted example of such a decision scheme is the mechanism regulating a central heating installation. Observation of the consequence of the action and the significance of this observation to the next decision are automatically built into the decision mechanism. Otherwise, the ordinary "trial and error" methods come into play. This decision scheme can normally also be used where action and reaction cover a long time, but it may then lead to substantial deviation. One can miss the target badly if information on the reaction to one's action is received too late. Furthermore, a great deal of time is lost before a correction can be applied. As a result, this decision mechanism loses its flexibility, and may lead to violent fluctuations. Finally, there are the decisions which are so long in taking effect as to be virtually made once and for all, whereby application of the "trial and error" method more or less amounts to making a random choice, and one has no alternative but to use the other model.

\section{The function of statistics in formulating economic policy}

Summing up, the theory provides a framework by which a decision problem can be quantitatively analysed, in which all the elements mentioned are given a place, and from which a decision can be taken using a criterion chosen beforehand. Hence, the theory does not relieve the decision-maker of his responsibility. It does not say what the decision is to be, but merely how it can be taken.

In this framework, statistics ${ }^{1}$ play a triple role: -

a. Statistics are used to mark out the field of operations. A general idea of the real situation to be affected by the decision is required before the problem can be formulated at all. The decision space and the limits of variation must be established. An approximate calculation must be made of the potential effects of nature, and one must be in a position to make the best possible estimate of the magnitudes of the variables of the utility function that will appear.

1 In this ariicle, "statistics" means the whole body of activities which result in quantitative data on a certain field becoming regularly available in the form of graphs and/or tables. 
b. Statistics must provide the data for the forecast model to be used in investigating the consequences of the various possible actions in the various possible circumstances. If necessary, it must supply the requisite figures for calculating the parameters of a forecast model (parameters of the equations which determine the relations between various relevant variables, parameters of the probability distributions which represent the uncertainties).

Here, statistics can only provide the basic material for a decision. This material will have to be analysed to transform it into the knowledge of facts required for the decision. The analytical methods used will differ greatly in accordance with the nature of the decision and the decision model chosen. It is therefore necessary to have fully competent analysts, as otherwise the figures cannot be used to the best advantage. Accordingly, there will have to be balance between statistical and analytical effort. The figures are, so to speak, the rough timber, which has to be dressed by the carpenter before it is fit for anything but firewood. However, a bad carpenter will never produce anything good, even if he uses the best wood in the world. This illustrates the function of the link which has to be established between economic policy and statistics, i.e. that of quantitative scientific analysis.

c. Finally, statistics must be regarded as one of the most important means of keeping a decision-making unit informed concerning developments after the decision has been taken.

If, therefore, a decision is looked at chronologically, statistics have a dual function. In the first place, it must provide the quantitative data for the forecast model (phases $a$ and $b$ ), and secondly it must measure the consequences of a decision already taken (phase c), in order to provide new data for the next decision. Statistics are thus a vital element in decision procedure.

When discussing the complication "time" in the decision model we saw that two different models are at our disposal, depending on the time horizon of the decision. For short-term decisions, marked by a rapid succession of action and reaction, the feed-back system can be used. Here the role of statistics will be mainly that described under c., i.e. to be a means of checking developments after a decision. The statistical information is necessary for the feed-back. In the analysis of longer-term decisions, the function of statistics is mainly that described under b., i.e. to produce more quantitative information for the forecasts.

\section{The economic aspect of statistics}

In economic terms, statistical data are a scarce commodity. Their collection costs money. Scarce production factors, in the form of labour and material, have to be sacrificed in order to obtain these data, and hence a cost curve can be adopted for them. The curve is, generally speaking, influenced chiefly by two factors, namely: a. the number and scatter of the units to be investigated,

b. the intensity of the investigation, i.e. the number of data to be collected per unit.

Accordingly, as soon as statistical information is required, a purely economic problem of allocation arises: how much money is to be spent, and - especially in the case of more complicated statistical systems, how are these costs to be distributed among the many possibilities of application? For instance, is one to work with samples investigated in great detail, or is a full census to be made with less detail in investi- 
gation? The first alternative emphasizes quantity at the expense of quality; the second does the opposite.

The situation can be likened theoretically to a market for information. The demand proceeds from the policy-making organs, which have to have the data in order to lay down and implement their policy. Like the demand for any other commodity, this demand concerns two properties, the quantity and the quality of the item desired, quality being, in this case, reliability. The price the customer is prepared to pay for the data depends on the advantage he expects to get from the better decisions resulting from better information. This profit can be more or less measured quantitatively by means of the decision models described in Section 2, using the following simple procedure. In the case of each decision, it is necessary to see what the result will be in the most favourable and the most unfavourable situation that can arise. The difference between these two must be set against the costs incurred in acquiring knowledge of the facts. For fuller knowledge of these facts will often make it possible to forecast the true situation more accurately, and this will enable the difference between the most favourable and the most unfavourable situation to be reduced.

The commodity is supplied by the statistical organizations, which should quote a kind of contract price, for which they can supply the information desired.

A system along these lines is, in fact, found in industry, where practically everything can be expressed as money. There is, for instance, a whole body of theory concerning checks on buying and selling and quality control throughout the production process. For example, in very many chemical works production is constantly under control by sampling. These checks are paid for by the improved quality of the product and the reduced losses. Budgeting and planning have introduced a certain equilibrium between investment in statistical services and the savings made thanks to these services. As regards government activities, this problem of allocation is far from having been solved.

By a process of over-simplification, the situation could be described as follows. The demand comes from economic policy-making organs, which frequently do not define their wishes further than by indicating the field on which they require information. They hardly touch the question of how refined the analysis should be, let alone construct models in which the statistical material supplied to them can be fully utilized and a clear connection established between knowledge of the facts and the usefulness of this knowledge.

Moreover, the costs of statistics are generally not paid by the policy-making organs, since the statistical office usually figures on another budget. Hence, the organs are not forced to weigh the utility of the information against its price. As a rule Ministries of Finance, with which responsibility for the budgets of statistical offices generally lies, judge by criteria quite different from that of the usefulness of the information, such as growth of the statistical office compared with previous years, growth of other government departments, and, most important of all, growth of the total State budget.

Finally the supplier of information, i.e. the statistical office itself, is allowed great freedom in apportioning the money extracted from the Ministries of Finance. Although most countries have machinery for confronting supply of statistical information with demand, it is not effective enough to maintain a close connection between economic policy and statistics; this means that in many countries statistics are published more out of habit or personal interest than because of their usefulness, or serve as padding in reports. 
Our theoretical answer to the problem of allocating the cost of statistics on economic policy problems will often remain theoretical, owing to the intricacy of the decision structure applied and the impossibility of fully expressing this cost quantitatively, as can frequently be done in industry. Nevertheless, it seemed expedient to include in our analysis the theoretical decision model described above, because it at any rate poses the question qualitatively, and so can help towards a better understanding of the fundamental allocation problem facing every statistical unit. To recapitulate: it is the decision-maker's utility function which determines the ultimate value of the statistics; the statistics must be worth the money spent on them: what facts must be observed and, more particularly, with what degree of precision, is a matter to be decided not by the statistician but by the decision-maker, after he has compared costs and utility. The statistician's duty is to provide the information requested as efficiently as possible.

\section{Examples}

In order to make all this clearer to the reader, a number of fictitious examples have been chosen in ascending order of intricacy, which indicate the lines of thought pursued and the difficulties to be surmounted in putting theory into practice.

The first two possible degrees of uncertainty in the decision problem will not be considered here. The situation of certainty is known to everyone who has ever had to choose from many possibilities in buying an article. For our purpose the games theory situation is not very interesting either, because it does not often arise as a zero-sum game in the field of economic policy. The game of negotiating is frequently much more complicated, and so far decision-making theory has yielded no solution whatever for it.

\section{Example 1}

The Government is considering control of a certain cattle disease by inoculation. However, manufacture of the vaccine takes time. By using our decision model, the quantity of vaccine required can be determined as follows.

The decision space consists in the different amounts of vaccine which can be ordered. The situation consists in a number of possible sizes of the cattle stock. The utility function is asymmetric. If there is too little vaccine, the disease may go on spreading for a certain time in some animals, because these animals cannot be inoculated and consequently may remain sources of infection. But if too much vaccine is ordered money is lost, especially if the vaccine will not keep. The first risk is obviously much more serious than the second. To put it simply, the losses when there are a thousand doses of vaccine too few will be many times as heavy as the cost of a thousand doses too many.

There is an element of uncertainty because the head of cattle for the following year has to be forecast from the census for this year. Three cases are possible: -

a. Account is taken only of the fact that the maximum annual fluctuation in the head of cattle is $5 \%$, according to a census carried out every year; but no account is taken of the probability distribution of these fluctuations. Accordingly, three situations can be postulated, viz.: -

1. Head of cattle next year $5 \%$ lower than this year,

2. Head of cattle next year same as this year,

3. Head of cattle next year $5 \%$ higher than this year. 
The decision space can be simplified by foreseeing three different decisions; to meet case 1, vaccine is ordered for $0.95 \%$ of the present number of animals, and so on. We therefore have a $3 \times 3$ matrix, in which each of the three decisions yields an optimum result for one situation.

From the resulting pay-off matrix it is clear that preference will be given to decision 3, which meets the situation in which there are $5 \%$ more animals than last year. Here, the Government runs the risk of having $10.5 \%$ too much vaccine, but it is certain of not having too little, which might be the case if decision 1 were taken, where $10 \%$ of the animals might be left without inoculation. The decision chosen will depend on the selection criterion taken, which, in its turn, depends on the policymaker's readiness to run a risk.

b. The trend in head of cattle is extrapolated by using the data for the previous years. This makes it possible to give a probability distribution of the situations, e.g. to show that over the last 10 years the average increase in head of cattle was $2 \%$ with a coefficient of variation of $1 \%$. Accordingly, if the possible situations are still to be confined to three, it will be best to take these three: -

1. Head of cattle next year same as present number,

2. Head of cattle next year equal to 1.02 of present number,

3. Head of cattle next year equal to 1.04 of present number.

If the trend continues, there is a $95 \%$ chance that reality next year will lie batween the above limits. Again three decisions are chosen to meet the situations. For the same utility function a pay-off matrix results which considerably restricts the possible losses. Here, too, decision 3 will probably be chosen, but now with a much smaller loss. With decision 3 there is a $2.5 \%$ risk of losing up to $4 \%$ of vaccine costs, as opposed to a $2.5 \%$ risk under decision 1 of up to $4 \%$ of the animals not being inoculated. In other words, extrapolation has greatly reduced the margin of risk in the pay-off matrix. It also makes it possible to try to deduce a probability distribution for all possible situations, and then to calculate mathematically the expected utility of each decision in these situations. The decision with the highest expected utility is then the most suitable choice.

c. Finally, attempts can be made to shorten the time taken to manufacture the vac-

cine. If this could be reduced to one month, the decision models used in the two preceding examples would not be necessary. It would be sufficient to order $95 \%$ of the vaccine necessary for the head of cattle in the previous year's cattle census, and if this should prove insufficient additional supplies could be ordered in time.

In this case, therefore, the feed-back system in its simplest form can be applied. If the first decision is unsatisfactory, quick and effective action can be taken to correct matters and still achieve an optimum result.

Ex a m p 1 e 2

The Government is contemplating support for the marketing of a certain farm product, such as wheat or butter. In principle there are two ways of giving this support: -

a. A guaranteed price can be fixed and, when the real price falls below it, the difference can be made up to farmers at the end of the season;

b. The Government can offer to take all quantities which cannot be sold at the guaranteed price and try to sell them as soon as the market recovers, or to export or denature them.

If it is assumed that, for budgetary reasons, the Government wants to restrict its 
total support to a minimum, the problem takes the following form. For each of the two intervention measures described, a number of target prices can be laid down, e.g. four levels, involving eight possible decisions. The state of reality is determined by the supply and demand curves on the market, which can be considered as represented by supply and demand elasticities. The number of possible situations can be reduced by proceeding from a number of suppositions regarding these elasticities, e.g. a high, medium and low situation can be taken for each of them. In all, then, nine possible situations result. The utility function remains relatively simple, because the consequences of each combination of the given decisions and situations can be evaluated in money, and the function can be reckoned as being in inverse proportion to the subsidy to be paid. This example clearly shows that analytical methods begin to play a part in these questions. Elasticities are not measurable quantities, but must be deduced from statistics by estimation (time-series and/or cross-section analysis). Besides a statistical uncertainty element there is the analytical uncertainty element, which again is based on two quantities, viz. the uncertainty inherent in the problem (represented by the disturbance term in the estimating equations) and the uncertainty resulting from the estimator. The total uncertainty therefore divides into three factors, and these can obviously also influence the statistical effort, which itself only influences one of the three. Where a phenomenon shows very great intrinsic variation in time, it will require correspondingly great statistical effort to ensure regular information on it, because it will have to be measured very often. However, this very great statistical effort cannot do much to limit the total uncertainty in the whole problem. Moreover, quantities which develop very evenly in time will be more suitable objects for a bigger statistical effort, because the element of uncertainty in the analysis will usually be smaller and so it will be necessary to ensure that the statistical uncertainty element is not disproportionately large.

This example also shows that statistical bodies must look ahead. Parameters for econometric models often have to be estimated by using time series which must cover many years if the requisite number of observations is to be obtained. Measurement after the event just is not possible.

Exam ple 3

The Government is contemplating a structural plan on which to base its investment outlay on consolidation of holdings, farms, buildings, etc. in a certain area. The outlay will have to be made in the next two or three years. One of the first problems is that of optimum size of holding, because this fixes the combination in which land. capital and labour are to work together. It is assumed that such a holding can be run by one family. The decision space can be put at three sizes of holding, with a further division by the type of farm building on which the accent is to be laid, for instance buildings for arable farming, pig-keeping or dairy-farming. The following possibilities may result: -

20 acres with accent on pig keeping,

20 acres with accent on dairy farming,

50 acres with accent on arable farming,

50 acres with accent on dairy farming,

100 acres with accent on dairy farming,

100 acres with accent on arable farming.

The range of possible situations here is very difficult to define. In the first place, 
we are concerned with long-term investment. Any choice is binding for many years. Size of holding and lay-out of buildings can only be changed at great expense. For this reason, the feed-back type of decision model cannot be used here at all. The various situations relate to the entire period of the next 15 to 20 years, and can best be characterized by certain price forecasts for the types of production listed. In doing this, it will be necessary to start from long-term supply and demand expectations in the country concerned. The problem can be standardized by assuming a certain average price trend for all farm products over the period concerned. For instance, as regards pig prices, three cases can be assumed: pig prices show the same trend as all other farm products; or they rise more quickly, say by $20 \%$; or more slowly, and lag $20 \%$ behind. If this is also applied to the other types of production, 27 possibilities result. The consequences of each decision would then have to be ascertained by means of farm models (programme planning or linear programming), which would have to incorporate certain hypotheses about technological development. In establishing the utility function, the costs connected with the various decisions would have to be taken into account. A hundred acres of arable farmland would generally cost considerably less than twenty acres for dairy cattle. On the other hand, less easily measurable factors also play a part, such as growth of the minimum income which is still considered socially justified. For instance, one type of farm will be more suitable for guaranteeing a higher average income, and will therefore be able to hold its own with the other population groups in the incomes race for a longer time than another type of farm.

Once the problem has been standardized as described above, the question immediately arises whether a certain probability distribution in the price pattern can be applied. It has already been pointed out that supply and demand analyses can be made. The basic statistical data must be analysed by a team of scientists, who will have to carry out the demand analyses required, manipulate the farm models, etc.

Reconsideration of the role of statistics in this problem shows that the analysts have to bridge an even greater gulf between statistics and economic policy than in the previous examples. Statistics provide the background material on which the model is based; but a whole series of scientific investigations is necessary to transform this model into decision parameters. Some of the data required lie wholly or partly outside the sphere of agricultural statistics proper, for instance data on the demand for farm products, and the demand forecasts to be based on them. It is also clear that interpretation of such a model may become vague because statistics are wanted in too many fields, with too little indication of the degree of precision required.

However, modern techniques of calculating by means of electronic computers render it possible to make these extensive preparations for a decision on such problems at a cost with regard to statistics needed, which is not so high as to outweigh the advantages of better appreciation of the risk inherent in the decision.

\section{Conclusions}

This article is intended to stimulate discussion of the subject rather than to offer final solutions for our problems. Accordingly, its conclusions are general, and must be seen more as suggestions for future investigation than as principles for taking "safe" decisions.

Modern decision theory turns on the realization that the uncertainty involved in almost every decision must be specifically incorporated in the mechanism by which the 
decision is taken, and cannot be eliminated by using some probability distribution, because it can only be evaluated by the decision-maker. His utility function determines the consequences of the uncertainty. Neither the analyst nor the statistician is required to stipulate how much effort he should put into his task: this depends chiefly on the element of uncertainty itself. Policy-maker, analyst and statistician cannot work in water-tight compartments but must approach their task as a team if the supply of information concerning the element of uncertainty is not to go wrong.

Modern decision theory has provided a structure within which teamwork can function and where each member, though assigned his own job, can see and follow up the consequences of what he is doing. The attempt to tackle these problems by casting them in the form of the decision models described above - however vague the application of these models may be at the moment - is thus of value to all concerned.

The advantage of the models for the policy-making body - even if they are only a mental exercise - is that they enable the hazards of the various possible courses of action to be better assessed; in making its final choice, the body concerned is not misled into considering only a few courses of action under only one given set of circumstances but also examines the consequences that the selected course might entail if everything should turn out badly. This provides a much better idea of the variability of the whole problem. The policy-maker will often be encouraged to leave the beaten track and seek out alternatives other than the normal starting-point of one "state of nature" on which to base his decision.

The advantage of this framework for the analyst is that his analysis is directed towards a concrete aim and at the same time specifically takes into account the element of uncertainty in a forecast. The analyst's procedure hitherto has been conscientiously to pass on his ideas about this uncertainty as a standard deviation or other measure of dispersion. The recipient usually has no idea what to do with the latter, and regards it as a superfluous detail. For the sake of simplicity he takes the single forecast as his basis, so that the whole plan, as regards the very important factor of uncertainty, rests on an alarmingly narrow foundation. The decision model offers many more possible ways of carrying the element of uncertainty in the forecast from the investigating stage on into the decision stage.

The advantage of this approach for the statistician is that his contributions to the various parts of the model are re-assembled by the forecast analyst and are evaluated by the responsible policy-maker. In addition, the element of uncertainty in his measurement is placed against that resulting from analyses, and this in turn facilitates the better balancing of these uncertainties - all against the background of the influence exerted by these elements of uncertainty on the utility function of the policy-maker.

Future developments depend on each member of the team examining his own task and improving his contribution and, more particularly, on the establishment both in government bodies and elsewhere of the organizational framework within which such teamwork can really be achieved. 